\title{
Characterization of the bone using structural borne acoustic waves
}

Haydar Aygun (PhD)*, Lawrence Yule, and Chris Barlow (PhD)

\section{Introduction:}

- During childhood, more bone is added than is being taken away. During early adulthood, the amounts removed and added are the same. If however, more bone is removed than is being added, we have a condition called osteoporosis.

- Osteoporosis literally means 'porous bone' and describes a period of largely asymptomatic bone loss leading to skeletal fragility and increased risk of fracture.

- It is caused by hormonal imbalance (oestrogen \& testosterone) and long-term cortico-steroid use, by low bone mass, as well as a weakened structure.

- One in three women and one in five men over the age of 50 will break a bone attributed to osteoporosis. Second only to cardiovascular disease as a global healthcare problem (World Health Organisation).

- The aim of this work is to investigate the use of a structural borne acoustic wave technique and ultrasonic wave technique to characterise the bone.

- When normal and osteoporotic bones are subjected to vibration, the resulting detected responses have different shapes, different natural frequencies, and amplitudes.

Measurement set-up for composite bone:

Measurement set-up is given in Figure below.

- An impact hammer, PCB086C03, is used to generate structural borne acoustic waves. The response is detected by using an accelerometer, MME-KS901.100.

- Impact hammer and accelerometer are connected to signal conditioning units, DJB-VB/01, which fed data acquisition, NI-USB-4431, which is connected to a computer.

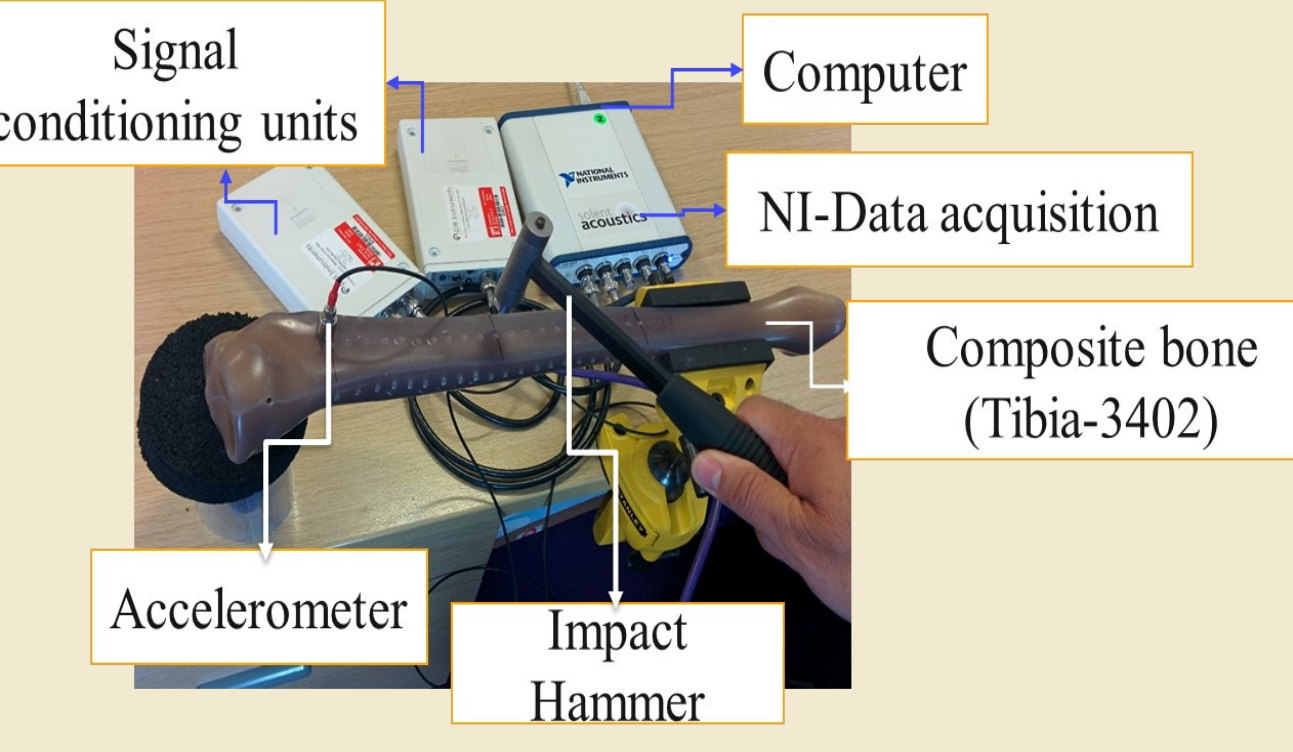

Transfer function of composite bone

- Detected responses using structural wave technique, can't be compared because initial forces applied to bone by an impact hammer are not repeatable.

- FFT has been used to convert filtered signals into frequency domain.

- Frequency response signals have been calculated by using transfer function method given as follows;

$$
T(f)=\frac{A(f)}{F(f)}
$$

- where $A(f)$ is the response of the system in frequency domain, and

- $F(f)$ is the force applied to system in frequency domain.
- Transfer function of the input and output signals detected at $10 \mathrm{~cm}$ along composite bone is given in Figure below.

- Increasing the perforation ratio of the composite bone changes the resonance frequency and the amplitude of the composite bones.

- Similar results can be observed at $20 \mathrm{~cm}$ along composite bone.

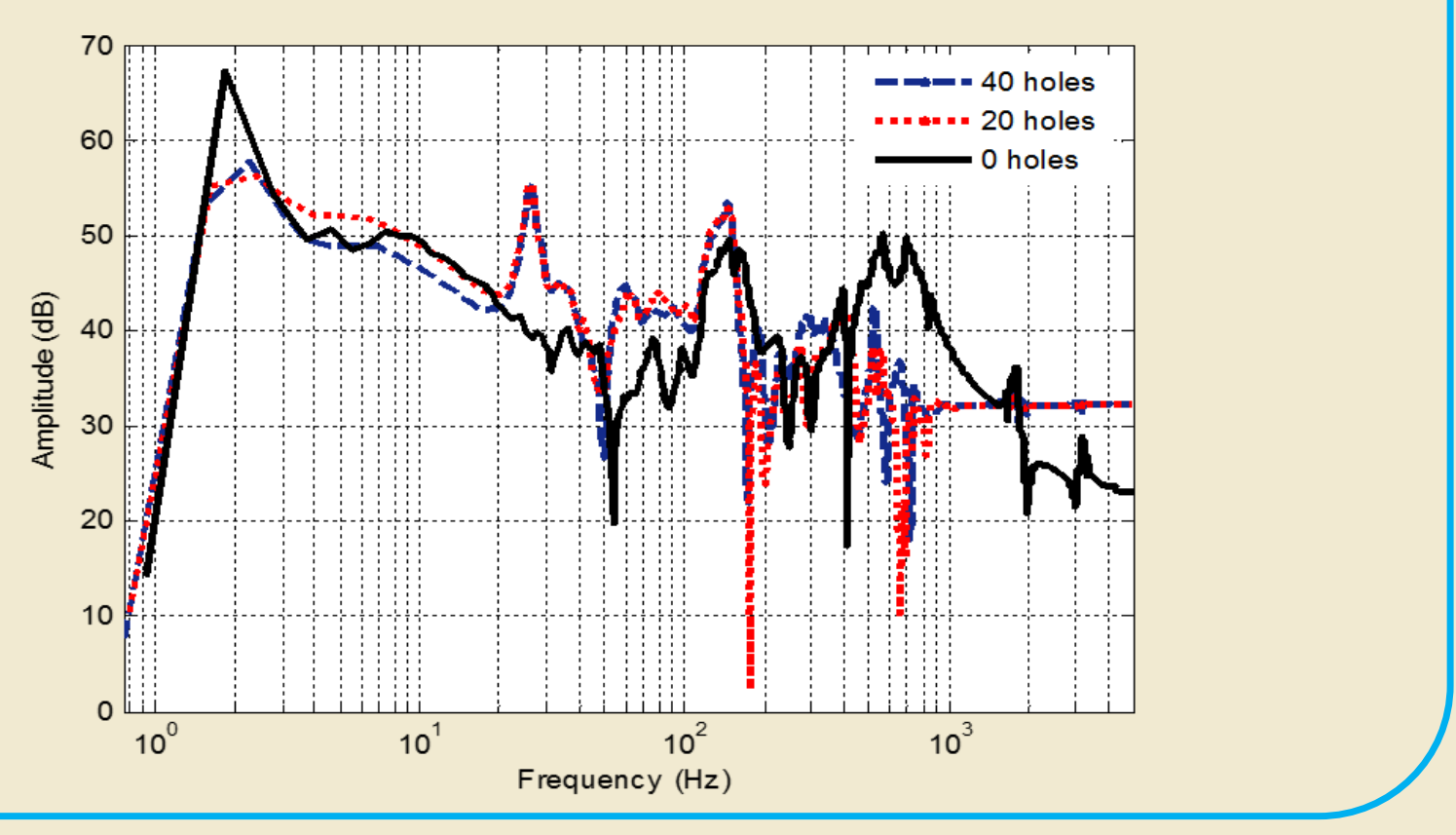

Measurement set-up for human bone (tibia):

- Measurement set-up for human tibia is given in Figure below.

- Input force has been applied to bone surface at five different locations along the bone, and the response has been detected under the knee cap on the surface of tibia for each measurements.

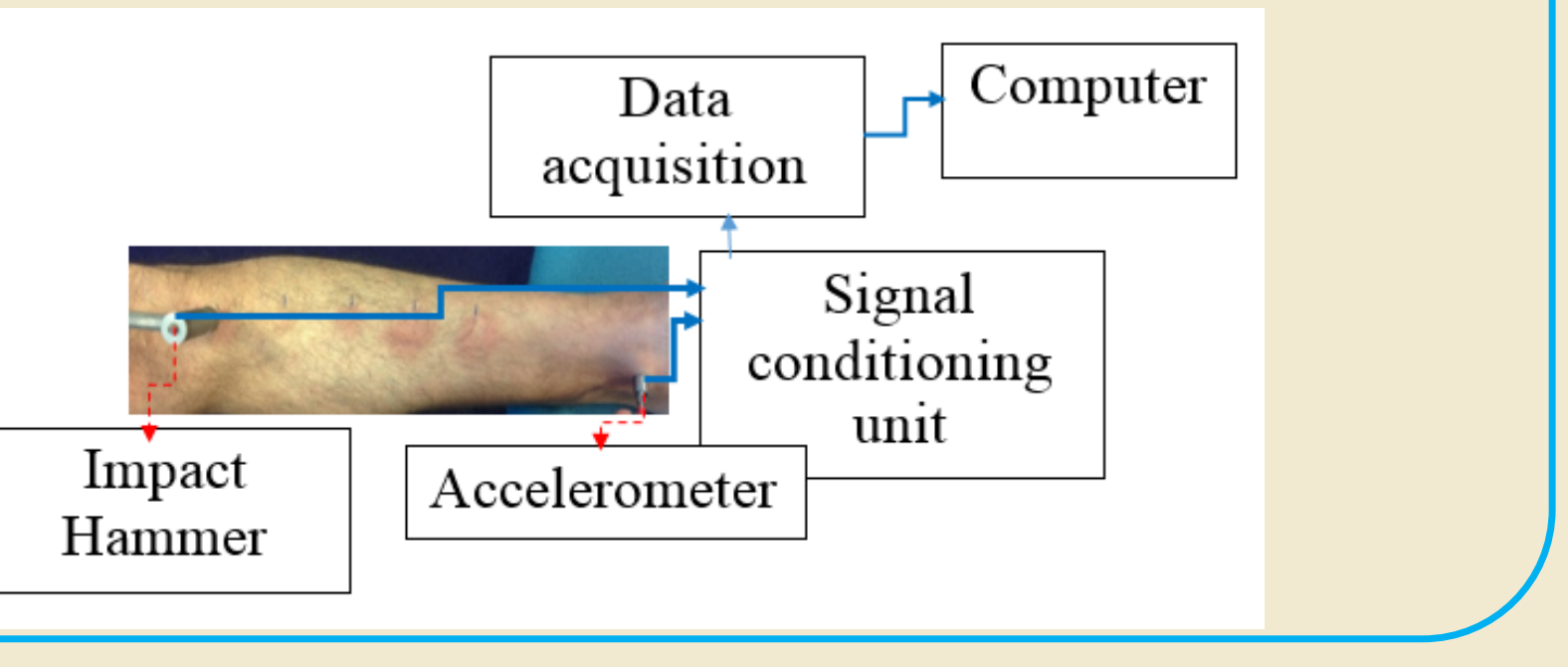

Transfer function of male tibia:

- Transfer function of a healthy young male tibia obtained under the knee cap at distance of 5 $\mathrm{cm}, 10 \mathrm{~cm}, 15 \mathrm{~cm}, 20 \mathrm{~cm}$ from accelerometer and ankle is shown in Figure below.

When the distance between the accelerometer and the impact hammer increases, the amount of structural borne acoustic energy transmitted through the bone mostly attenuates throughout the frequency range.

- This attenuation of acoustic energy might be due to the distance between input and outputs, soft tissue of the bone, or changes in bone structure and diameter along the tibia.

The natural frequency of the male tibia obtained under the knee cap at distance of $5 \mathrm{~cm}$, $10 \mathrm{~cm}, 15 \mathrm{~cm}$ and $20 \mathrm{~cm}$ from the accelerometer and ankle are given as $558.4 \mathrm{~Hz}, 285.2 \mathrm{~Hz}$, $294.4 \mathrm{~Hz}, 288.5 \mathrm{~Hz}$ and $127.1 \mathrm{~Hz}$ respectively.

- Increasing the distance between accelerometer and hammer causes the natural frequency of male bone to shift to lower frequencies.

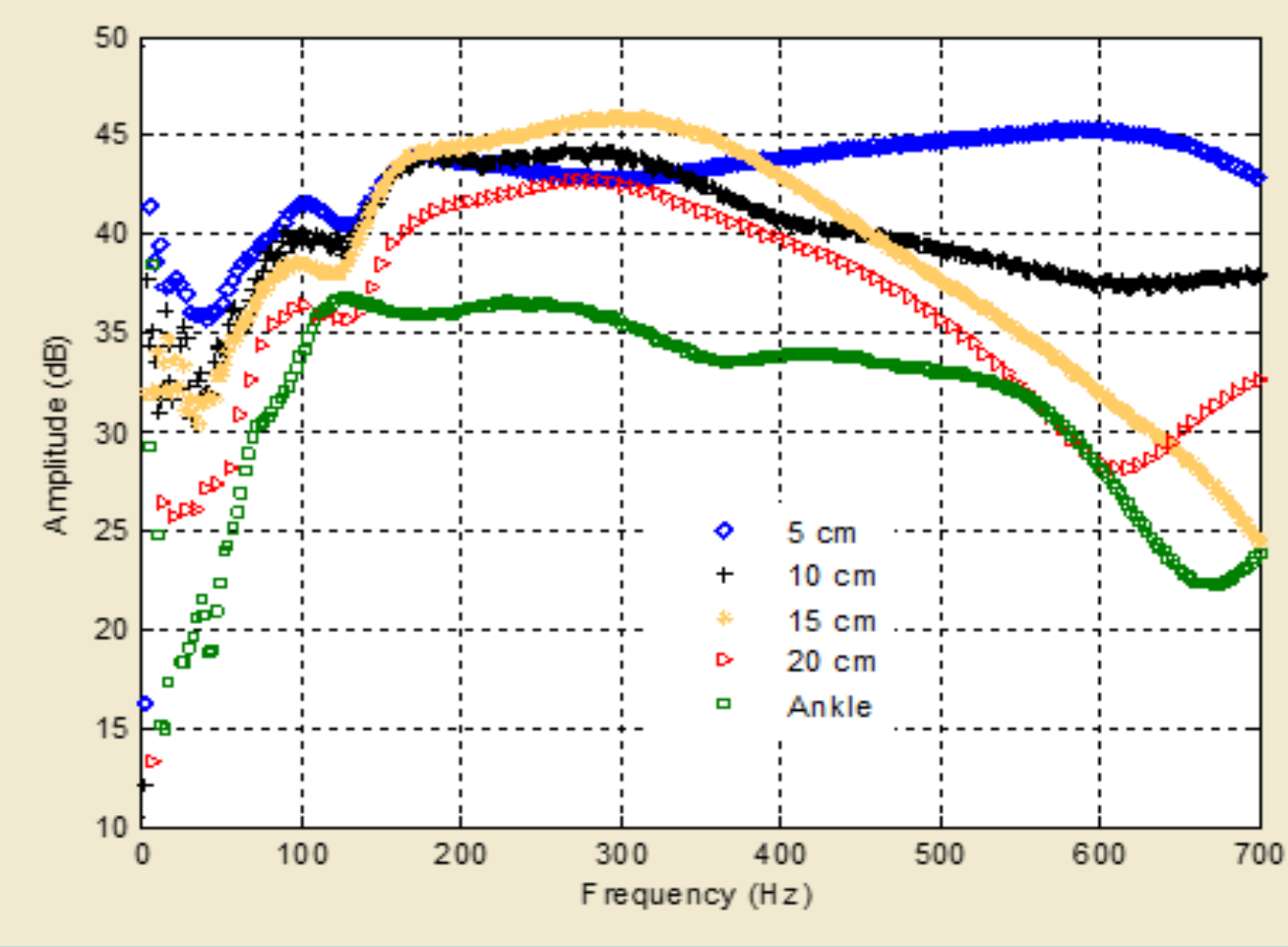

Transfer function of human (female) tibia:

- The natural frequency of female tibia obtained under the knee cap at distance of $10 \mathrm{~cm}$ and $20 \mathrm{~cm}$ from accelerometer are given as $126.7 \mathrm{~Hz}$ and $121 \mathrm{~Hz}$ respectively as shown in Figure below.

- The amplitude of natural frequency of female bone is between $36 \mathrm{~dB}$ and $44 \mathrm{~dB}$.

- More acoustic energy was attenuated throughout frequency range when the distance between accelerometer and impact hammer is increased.

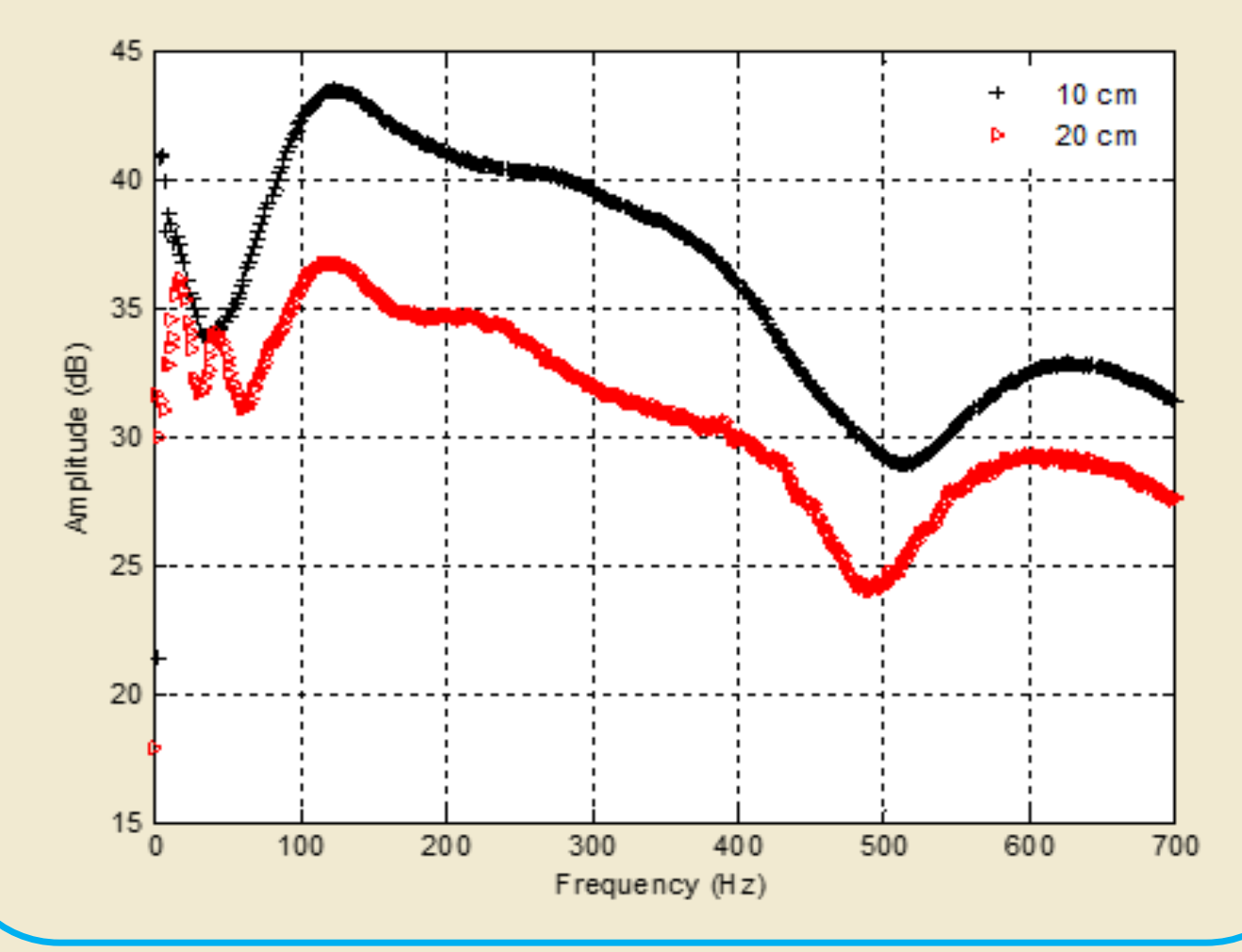

Conclusion:

- An experimental investigation has been carried out to determine the possibility of using a structural borne acoustic wave technique to detect the variation of sound propagation in the bone structure. Both tibias tested have different dimensions and size.

- Varying the distance between the accelerometer and impact hammer mostly changes the natural frequency of the male tibia and the amplitude of response while it slightly changes the natural frequency of the female tibia and reduced the amplitude of response a lot throughout the frequency range.

- The results show that tibia has an anisotropic structure which has an important effect on measured natural frequencies.

- Further work is needed to carry out measurements on more healthy and unhealthy male and female tibias.

\section{References:}

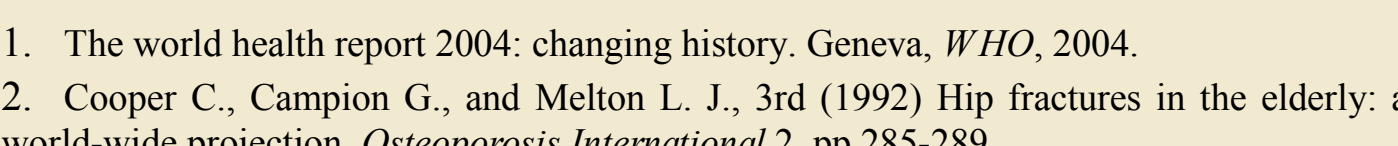

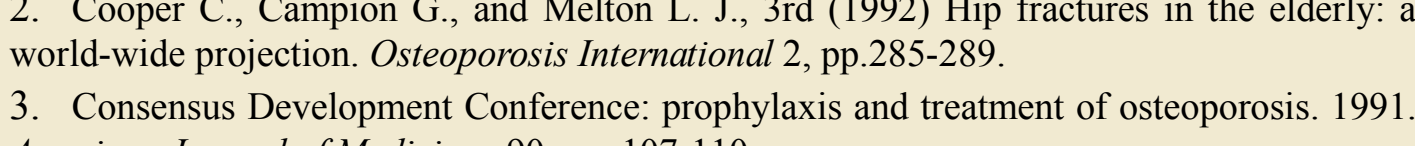

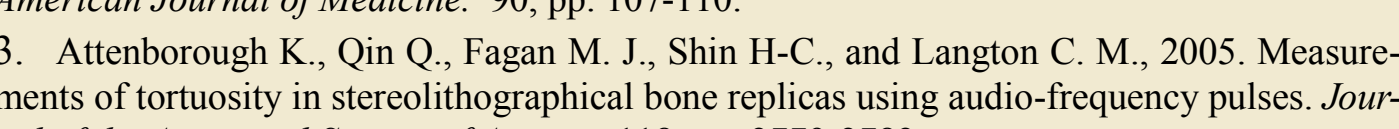
nal of the Acoustical Society of America 118, p. 2779-2782. 4. Ayguin $\mathrm{H}$, Attenborough $\mathrm{K}$., Lauriks $\mathrm{W}$., and Langton M.C., 2010, Ultrasonic wave
propagation in Stereolithographical bone replicas. Journal of the Acoustical Society of America 127 (6), pp. $3781-3789$.
5. Aygün H., Attenborough K., Lauriks W., Rubini P.A., and Langton M.C., 2011 , “Wave

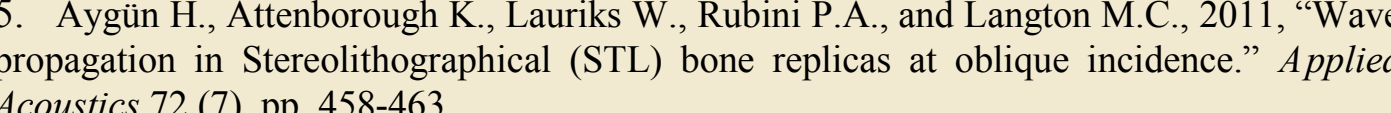
6. Ayguin $\mathrm{H}$., and Barlow C., 2015 , Ultrasonic wave propagation through porous ceramics
it different angles of propagation. Applied Acoustics 88 , pp. 6-11. Hans D and Kreig M A 2009. Quantitative ultrasound for
nent of osteoporosis. Saludpublica de Mexico 51, S25-S37. 